\title{
APPROPRIATE MODELS FOR SIMULATING OPEN-POROUS MATERIALS
}

\author{
Tomasz WeJrzanowski, Samih Haj Ibrahim ${ }^{\bowtie}$, JAKUb Skibinski, Karol CwiEKa \\ AND KRZYSZTOF JAN KURZYDLOWSKI
}

Warsaw University of Technology, Faculty of Materials Science and Engineering, Woloska 141 Str., 02-507

Warsaw, Poland

e-mail: twejrzan@inmat.pw.edu.pl, samih@vp.pl,j.skibinski@inmat.pw.edu.pl,karol.cwieka@inmat.pw.edu.pl

(Received October 17, 2016; revised March 21, 2017; accepted April 17, 2017)

\begin{abstract}
In the present paper two representative models applied for modeling of two types of porous materials - opencell foams and open porosity tapes - are addressed. Algorithms presented here base on Laguerre-Voronoi tessellations (open-cell foams) and the sphere representation (open-porosity tapes) and enable creating the desired porosity and pore size distribution. The geometrical features of the models, such as: porosity, mean pore size, cell diameter distribution and number of faces per cell were compared with those obtained by 3D micro-computed tomography and good agreement was obtained.
\end{abstract}

Keywords: open-porous, models, open-foams, $\mu \mathrm{CT}$.

\section{INTRODUCTION}

In recent years, applications of open-porosity materials have significantly increased. Energy absorbers, filters, bone implants, catalysts and heat exchangers are much more commonly made from open-porous materials. The rising popularity can be explained by their unique characteristics, whereby the porous microstructure is a major factor determining their properties and deciding on their applications. In particular: porosity, pore size distribution and specific surface area are of key importance for functional and structural material properties. To design optimal structures of materials, the structure-property relationships are analyzed. Numerical modeling is an useful tool, which facilitates the materials simulation process, when combined with fabrication and characterization techniques (Fig. 1).

All the simulations related with investigations of properties and/or processes require a proper model of the studied materials structure. Various models of open porosity materials have been applied in the literature. Foam structures are frequently described with use of geometrical models, based on the assumption of equal volume of pores of different shapes (Thomson, 1887; Zhu et al., 1997; Warren and Krayniki, 1997; Grenestedt, 1999; Christensen, 2000; Konstantinidis and Tsipas, 2010). However, experimental observations show that foams exhibit a distribution of pore sizes (Lautensack and Sych, 2006). Good approximation of such distributions can be obtained by using a Laguerre-Voronoi algorithm (Fan et al., 2004; Redenbach, 2009), whereby tessellations are performed on a set of spheres with pre-determined size distribution (Wejrzanowski et al., 2013). There are also a series of approaches in modeling of the openporous structures obtained by sintering of spherical particles. Many are based on simplified geometries, whereby the complex and stochastic structure is replaced by a more regular model that is easier to be described and computed, i.e., agglomerate model (Prins-Jansen et al., (1997; Lueth et al., 2016). Others include representations of the structural entities as a set of spheres (Gaiselmann et al., 2013) or metaball objects (Depczynski et al., 2016). The discrete element method is especially useful for modeling of the openporous structures (Stroeven et al., 2012). Numerical models obtained from computer tomography are also frequently used for studying the properties of commercial materials (Fiedler et al., 2009; Veyhl et al., 2011), however such an approach does not render it possible to simulate the materials.

In the present study, Laguerre-Voronoi tessellations (LVT) and sphere representation are described as a useful tool for the modeling and the simulation of open-cell foams and open-porosity tapes, respectively. The parameters for LVT were obtained based on $\mu \mathrm{CT}$ study of commercially available alumina foams. Geometrical features for the tape model were obtained by quantitative characterization of porous nickel based tapes for Molten Carbonate Fuel Cell (MCFC). 


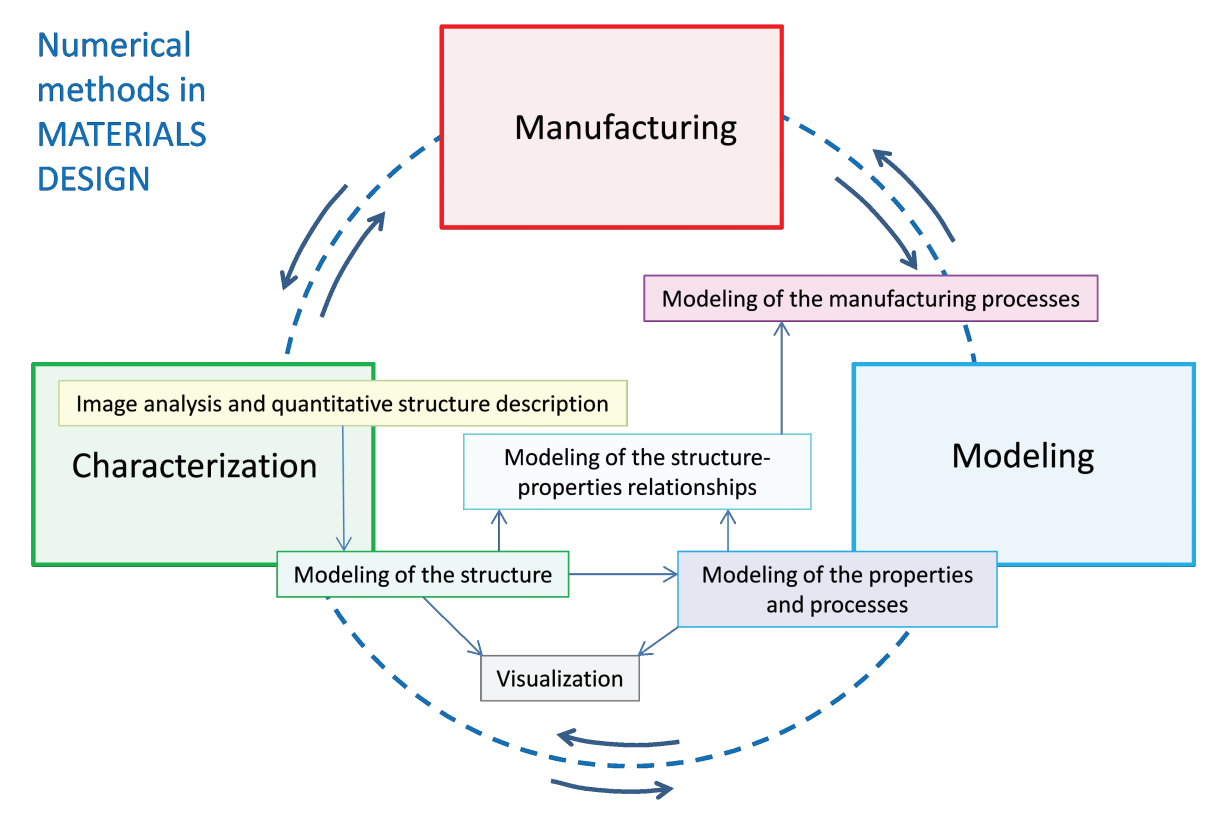

Fig. 1. Schematic illustration of the numerical methods in material design concept.

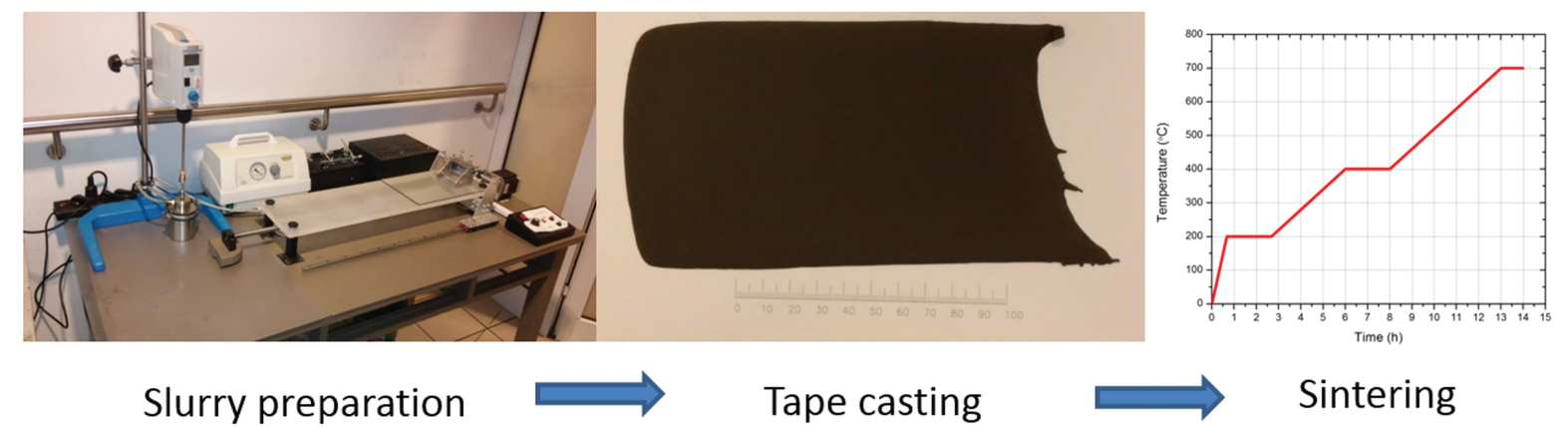

Fig. 2. Scheme of the fabrication process of the open-porous samples.

\section{MATERIALS AND METHODS}

\section{REFERENCE MATERIALS}

In the studies presented herein, two different types of porous materials were used as the reference for design and validation of the models. First materials relevant to particle-based porous structure were not commercially available and were fabricated within this studies by tape casting process. The fabrication process consists of 3 stages (Fig. 2). In the first step, slurry for casting of green tapes was obtained by mixing metallic (nickel) powders with polymeric binder, water and other additives (i.e., dispersant). Then the slurry was casted on the moving glass surface through the $1 \mathrm{~mm}$ gap in the doctor blade. In the last stage, material is burned out in the reducing temperature $\left(\mathrm{N}_{2}+\mathrm{H}_{2}\right)$. That process resulted in thermal decomposition of organic compounds and open-porous structure was obtained. Four samples were prepared for further studies. Due to MCFCs being still at the level of scientific research, there are no commercially available materials for reference. The second material represented foam-like structure. In this case the commercial ceramic foam filters manufactured with polymeric sponge method were used. This method produces open-cell structures by impregnating a polymeric sponge with a ceramic slurry, which is then burned out to leave a porous ceramic. Reference structures used in this study were commercially available aluminum foam. 3 specimens with nominal pore density of 0.39 pores per $\mathrm{mm}$ (commercial designation 10 pores per inch) were employed.

\section{CHARACTERIZATION}

Open-porous materials were characterized with use of micro-computed tomography $(\mu \mathrm{CT})$. High resolution X-Radia XCT-400 equipment was used, under acceleration of $50 \mathrm{kV}$ and current $10 \mathrm{~A}$, which provided final resolution of $1.1 \mu \mathrm{m}$ per 
voxel. Procedure of image reconstruction, processing and binarization was described in previous work (Regulski et al., 2015). Quantitative image analysis was performed for both $\mu \mathrm{CT}$ and representative models, using SkyScan CTanalyser software. The following parameters were examined in order to characterize porous structure of samples:

- Porosity $\left(V_{V}\right)$ - the ratio of pore volume to the total volume

- Specific surface area $\left(S_{V}\right)$ - the ratio of total solid surface to the total volume

- Mean pore size $(E(d))$ - the average diameter of spheres inscribed in the pore space

- Mean strut size $(E(l))$ - the average diameter of spheres inscribed in the solid space.

\section{MODELING}

\section{Particle based open-porous structure model}

The process of modeling of the open-porous material structure (see Fig. 3) was initiated by generation of polydispersive spheres, with the same size distribution as nickel powder particles used to manufacture real electrode materials. Spherical shape of powder particles was assumed. Then, spheres were iteratively packed in a box with the smallest possible size, by random movement of the spheres, with non-overlapping condition and simultaneous decrement of the box size (Wejrzanowski et al., 2013), also known in the literature as random close packing (RCP) algorithm. Next step was conducted in ANSYS Mechanical software with APDL script, which generated open-porous structure, by removing selected particles. Spheres were enlarged in order to achieve intersection, which was necessary for the continuity of the structure. To reflect statistical character of the materials structure obtained by tape casting method, spheres to be retained were selected randomly, starting from a set number of arbitrary points. Next, randomly picked spheres were retained if there was intersection with already drawn spheres. As a result, structure growth from chosen points was obtained. To summarize, there were four parameters which controlled generation of the structure of the model: starting number of keypoints, size distribution of the spheres, number of spheres to be kept and magnification of the spheres. Each of these parameters was fitted after comparison of the model with real material structure, so the discrepancy of the analyzed structural parameters between real material $\mu \mathrm{CT}$ images and model structures was minimized. Three structures were generated for each set of parameters with size comparable to the $\mu \mathrm{CT}$ images $(100 \mu \mathrm{m}$ cube).
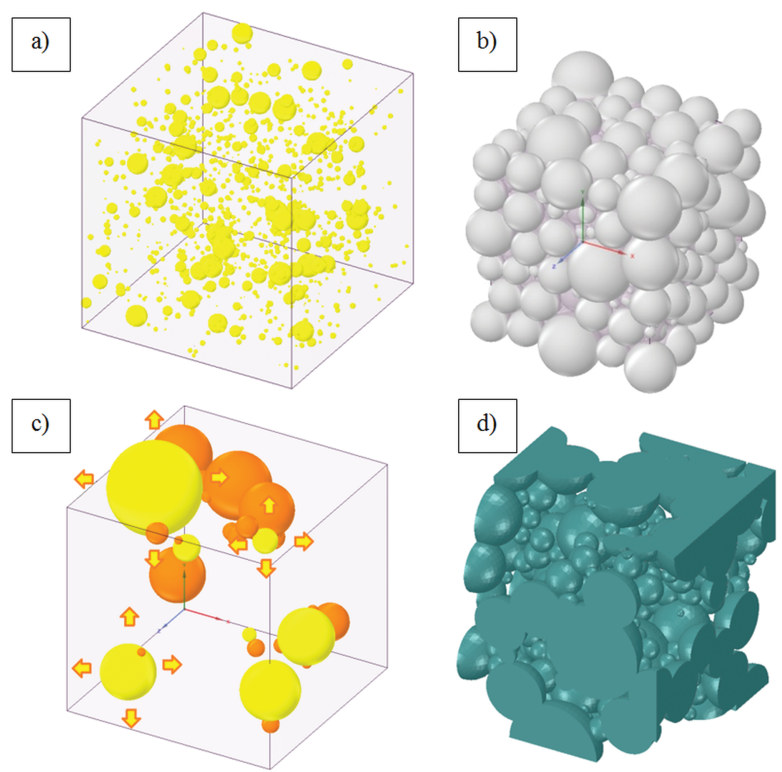

Fig. 3. Scheme of structure building. a) generation of spheres, b) compression, c) Structure growing from starting points and d) final structure.

\section{Foam structure model}

The design algorithm developed to model foam structures is based on the Laguerre-Voronoi tessellations method (Wejrzanowski et al., 2013; Skibinski et al., 2015; 2012). Tessellations are applied to a set of spheres, with specified size distribution, packed in a cube. The foam struts were generated by creating cylinders with defined radius along the cell edges. The algorithm for the modeling of foam structures employed in the present study consists of the following five steps:

1. Generation of spheres with a defined size distribution (in the computation log-normal distribution of volume was adopted with a pre-selected average value, and the variation coefficient)

2. Packing of the spheres,

3. Performing Laguerre-Voronoi tessellations,

4. Defining the triple edges of the cellular structure, and

5. Generation of struts along the triple edges.

The first two steps are performed with use of the same software as in case of modeling porous tapes. This modelling procedure can be used to 
study the influence of geometrical parameters on various properties such as permeability, mechanical properties, heat transfer properties or gas species volume fraction distribution on MCFC anode. The algorithm is adapted individually to the reference or commercial materials, depending on the studied issue. Parameters such as strut thickness, mean pore size, specific surface or number of pores are chosen individually for each application. In the present study, three random structures with homogeneous pore size distribution were generated using the procedure described above. Homogeneity (or inhomogeneity) of the structure can be expressed as coefficient of variation, which is the ratio of standard deviation and the mean value of spheres diameters $E(d)$ or pore volumes $E(V)$. The coefficient of variation, calculated based on the diameters of the spheres used in the design procedure $(\mathrm{CV}(d))$, was set to be 0.1 , which corresponds to material with the most homogeneous pore size distribution. Resulting coefficient of variation of pore volumes $(\mathrm{CV}(V))$ was $0.458,0.478$ and 0.484 . The difference in the obtained values of $\mathrm{CV}(V)$ results from random nature of the design procedure, each generated structure is slightly different. Moreover, differences between the obtained values is quite small, since very inhomogeneous structures have $\mathrm{CV}(V)$ up to 2 . Each structure was limited by a bounding box of cubic shape and consisted of 200 pores. Strut diameter of $1.5 \mathrm{~mm}$ was chosen based on the mean strut diameter of the reference structures. Such strut diameter resulted in porosity ranging from 81.4 to $83.6 \%$. Micro-computed tomography was used to investigate structural parameters of three different samples of reference materials with the same commercial designation of 10 ppi.

Table 1. Results of image analysis of the open-porous samples after fitting.

\begin{tabular}{cccc}
\hline & $E(d)$ & $S_{V}$ & $V_{V}$ \\
& $\mu \mathrm{m}$ & $1 / \mu \mathrm{m}$ & $\%$ \\
\hline$\mu$ CT model & 10.49 & 0.199 & 46.93 \\
Standard Deviation & 0.78 & 0.006 & 2.27 \\
\hline Representative model & 10.67 & 0.212 & 48.05 \\
Standard Deviation & 1.45 & 0.004 & 2.12 \\
\hline Difference & $1.7 \%$ & $6.5 \%$ & $2.4 \%$ \\
\hline
\end{tabular}

\section{RESULTS}

Obtained tape samples had a good cohesion and cross sectional images of the material revealed open-porous structure (see Fig. 4a). The 3D $\mu \mathrm{CT}$ and representative models have been shown in
Fig. 4. Similar pore structure can be observed despite the fact, that the representative model consists of regular spheres. Geometrical features (porosity, specific surface area and mean pore size) of the representative model after fitting are compared with parameters of real material in Table 1 . The difference of obtained values between $\mathrm{CT}$ and representative models does not exceed $10 \%$.

Example of foam filter, digitalized model obtained with $\mu \mathrm{CT}$ and the designed foam are shown in Fig. 5. Complete set of structural parameters of both designed and commercial materials is listed in Table 2. The total scanned volume was related to the scanning resolution. Scans were performed with resolution of $45 \mu \mathrm{m}$ and resulted in a cubic shaped geometry of $35 \mathrm{~mm}$ edge length. Such resolution is sufficient for obtaining precise representation of complex foam geometry, and the obtained sample size seems sufficient to be treated as representative regarding the mean pore size of foams, since literature indicates from 5 to 10 pores per edge length of volume to be treated as representative (Roberts and Garboczi, 2002; Veyhl et al., 2011). However for structures with inhomogeneous pore size distribution representative sample size will increase, and larger structures should be considered.

\section{DISCUSSION}

In the present study a methodology for generation of models of the porous structures has been presented. The spherical model of porous structure exhibits geometrical features similar to the ones of manufactured tapes. The negligibly small discrepancy proves that our model is a good representation of the tape casted open-porous materials and can be used in further research of a structure-properties relationships as well as for structure optimization process for a given application. The LVT method, on the other hand, is a useful tool for design of open-cell foam structures, as the parameters of modeled structures are in accord with parameters of studied foam filters. Obtained structural parameters of commercial foam materials are in good agreement with literature data (Große et al., 2009; Veyhl et al., 2011; Fiedler et al., 2012). The porosity of designed structures seem slightly higher than in case of foam filters. This fact is caused by clogged pores of commercial materials, which are the effect of the production process. Image analysis of smaller areas of the material without clogged pores exhibit up to $2 \%$ higher porosity. Additionally struts of commercial materials are thicker at the triple junctions. This effect is not included in the LVT model. It was shown that models created are representative and can 


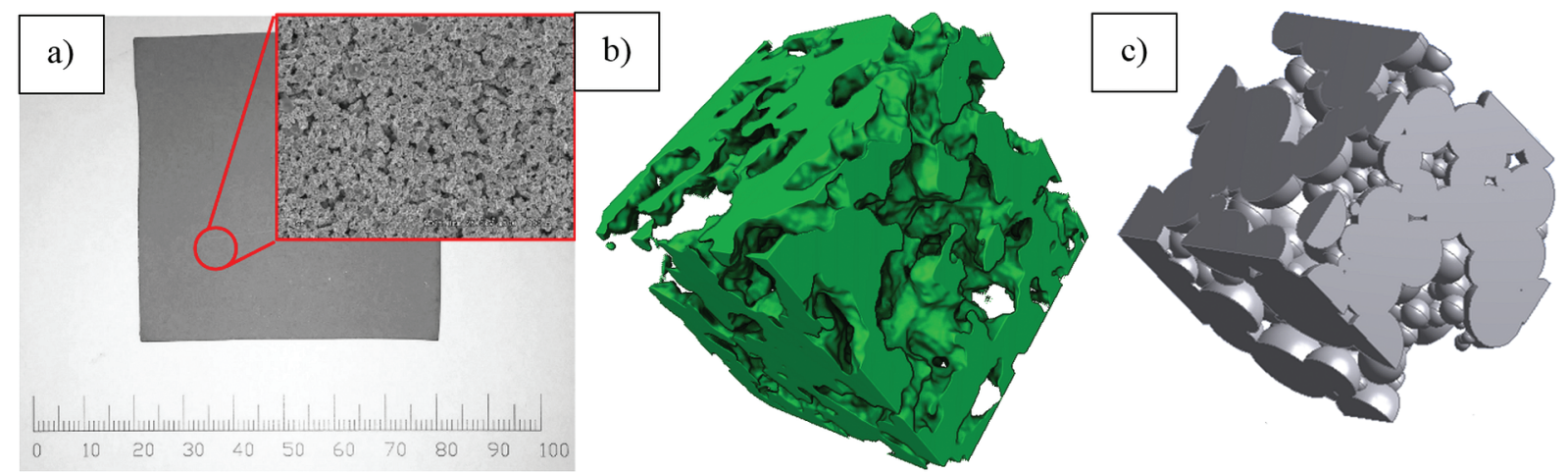

Fig. 4. a) Real electrode material, b) 3D $\mu$ CT image and c) model of open-porous material.
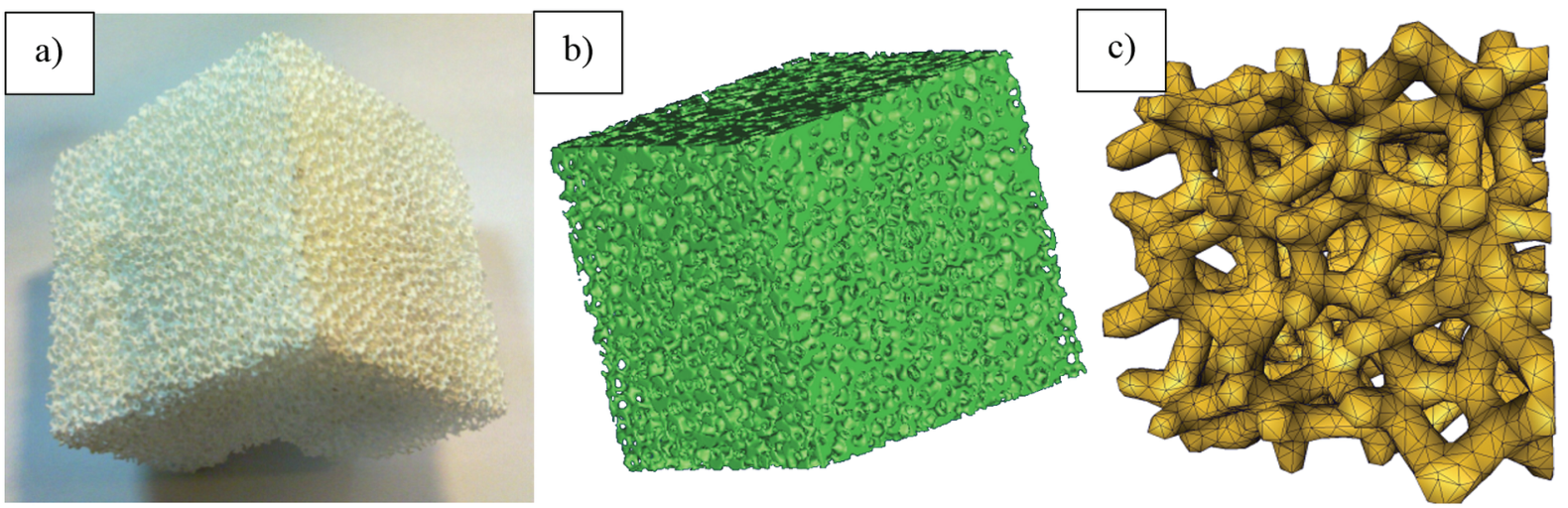

Fig. 5. a) Real foam element, b) 3D $\mu C T$ image and c) model of open-cell foam.

Table 2. Structural parameters of LVT structures and commercial foam materials.

\begin{tabular}{|c|c|c|c|c|c|c|c|}
\hline \multicolumn{2}{|c|}{ Structure } & 10ppi & 10ppi & 10ppi & LVT & LVT & LVT \\
\hline$V_{V}$ & [\%] & 78.30 & 77.02 & 78.96 & 81.40 & 81.54 & 83.61 \\
\hline$E(d)$ & {$[\mathrm{mm}]$} & 2.56 & 2.56 & 2.56 & 3.19 & 3.10 & 3.01 \\
\hline$E(l)$ & {$[\mathrm{mm}]$} & 1.43 & 1.39 & 1.45 & 1.5 & 1.5 & 1.5 \\
\hline$E(V)$ & {$\left[\mathrm{mm}^{3}\right]$} & 8.78 & 8.78 & 8.78 & 16.99 & 15.60 & 14.28 \\
\hline$S_{V}$ & {$[1 / \mathrm{mm}]$} & 696.45 & 705.12 & 680.74 & 577.91 & 575.41 & 571.77 \\
\hline
\end{tabular}


be applied for further research.The described findings render possible the application of the described design methods for studying the influence of various structural parameters, such as pore size distribution, strut shape or pore diameter, on utility properties such as Young's modulus, Poisson's ratio, permeability or heat transfer properties.

\section{ACKNOWLEDGMENTS}

This work was supported by the National Center for Research and Development in the framework of the "Blue Gas Program" (Grant No. BG1/IRES/2013).

\section{REFERENCES}

Christensen RM (2000). Mechanics of cellular and other low-density materials. Int J Solids Struct 37:94-104.

Depczynski W, Kazala R, Ludwinek K, Jedynak K (2016). Modelling and microstructural characterization of sintered metallic porous materials. Materials 9(7):567.

Fan Z, Wu Y, Zhao X, Lu Y (2004). Simulation of polycrystalline structure with Voronoi diagram in Laguerre geometry based on random closed packing of spheres. Comput Mater Sci 29:301-8.

Fiedler T, Solorzano E, Garcia-Moreno F, A. Öchsner, Belova IV, Murch GE (2009). Computed tomography based finite element analysis of the thermal properties of cellular aluminium. Mater Sci Tech Ser 40:139-43.

Fiedler T, Belova IV, Murch GE (2012). $\mu$ CT-based finite element analysis on imperfections in open-celled metal foam: Mechanical properties, Scripta Mater 67:455-8.

Gaiselmann G, Neumann M, Holzer L, Hocker T, Prestat MR, Schmidt V (2013). Stochastic 3D modeling of $\mathrm{La} 0.6 \mathrm{Sr} 0.4 \mathrm{CoO} 3$ cathodes based on structural segmentation of FIB-SEM images. Comput Mater Sci 67:48-62.

Grenestedt JL (1999). Effective elastic behavior of some models for perfect cellular solids. Int J Solids Struct 36:1471-501.

Große J, Dietrich B, Garrido GI, Habisreuther P, Zarzalis N, Martin H, Kind M, Kraushaar-Czarnetzki B (2009). Morphological characterization of ceramic sponges for applications in chemical engineering. Ind Eng Chem Res 48(23):10395-401.

Konstantinidis ICh, Tsipas SA (2010). Symmetry effects and their influence on the mechanical behavior of open and closed cell Al foams. Mater Des 31:4490-5.
Lautensack C, Sych T (2006). 3D image analysis of open foams using random tessellations. Image Anal Stereol 25:87-93.

Lueth S, Sauter US, Bessler WG (2016). An agglomerate model of lithium-ion battery cathodes. J Electrochem Soc 163(2):A210-22.

Prins-Jansen JA, Hemmes K, De Wit JHW ((1997). An extensive treatment of the agglomerate model for porous electrodes in molten carbonate fuel cells I. Qualitative analysis of the steady-state model. Electrochim Acta 42(23):3585-600.

Redenbach C (2009). Microstructure models for cellular materials. Comput Mater Sci 44(4):1397-407.

Regulski W, Szumbarski J, Wollk-Laniewski L, Gumowski K, Skibinski J, Wichrowski M, Wejrzanowski T (2015). Pressure drop in flow across ceramic foams - A numerical and experimental study. Chem Eng Sci $137: 320-33$.

Roberts AP, Garboczi EJ (2002). Elastic properties of model random three-dimensional open-cell solids. J Mech Phys Solids 50:33-5.

Skibinski J, Cwieka K, Kowalkowski T, Wysocki B, Wejrzanowski T, Kurzydlowski KJ (2015). The influence of pore size variation on the pressure drop in open-cell foams. Mater Design 87:50-5.

Skibinski J, Wejrzanowski T, Szumbarski J, Kurzydłowski KJ (2012). Computational design of the flow properties of foams. WIT T Eng Sci 74:109-18.

Stroeven P, Le LBN, Sluys LJ, He H (2012). Porosimetry by random node structuring in virtual concrete. Image Anal Stereol 31:79-87.

Thomson W (1887). On the division of space with minimum partitional area. Philos Mag 24(151):503-14.

Veyhl C, Belova IV, Murch GE, Fiedler T (2011). Finite element analysis of the mechanical properties of cellular aluminium based on micro-computed tomography. Mater Sci Eng A Struct 528(13-14):4550-5.

Warren WE, Krayniki AM (1997). Linear elastic behavior of a low-density Kelvin foam with open cells. J Appl Mech 64:787-94.

Wejrzanowski T, Skibinski J, Szumbarski J, Kurzydlowski KJ (2013). Structure of foams modeled by LaguerreVoronoi tesselations. Comput Mater Sci 67:216-21.

Zhu HX, Knott JF, Mills NJ (1997). Analysis of the elastic properties of open-cell foams with tetrakaidecahedral cells. J Mech Phys Solids 45:319-43. 